\title{
BMJ Open Qualitative interviews with healthcare staff in four European countries to inform adaptation of an intervention to increase chlamydia testing
}

Cliodna McNulty, ${ }^{1}$ Ellie J Ricketts, ${ }^{2}$ Hans Fredlund, ${ }^{3}$ Anneli Uusküla, ${ }^{4}$ Katy Town, ${ }^{5}$ Claire Rugman, ${ }^{1}$ Anna Tisler-Sala, ${ }^{4}$ Alix Mani, ${ }^{6}$ Brigitte Dunais, ${ }^{6}$ Kate Folkard, ${ }^{5}$ Rosalie Allison, ${ }^{1}$ Pia Touboul ${ }^{7}$

To cite: McNulty C, Ricketts EJ, Fredlund $\mathrm{H}$, et al. Qualitative interviews with healthcare staff in four European countries to inform adaptation of an intervention to increase chlamydia testing. BMJ Open 2017;7:e017528. doi:10.1136/ bmjopen-2017-017528

- Prepublication history and additional material for this paper are available online. To view please visit the journal (http:// dx.doi.org/10.1136/bmjopen2017-017528).

Received 27 April 2017 Revised 14 July 2017

Accepted 4 August 2017

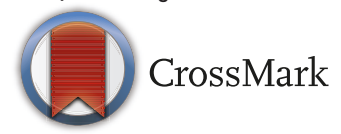

${ }^{1}$ Department of Microbiology,

Public Health, Primary Care Unit, Gloucestershire Royal Hospital, Derriford Hospital, Plymouth, UK ${ }^{2}$ Derriford Hospital, Plymouth, UK ${ }^{3}$ Department of Laboratory Medicine, County Medical Officer, Orebro University, Orebro, Sweden

${ }^{4}$ Department of Family Medicine and Public Health, University of Tartu, Tartu, Estonia

${ }^{5}$ Department of HIV and STI, Centre for Infectious Disease Control and Surveillance, Public Health England, London, UK ${ }^{6}$ Departement of de Sante Publique, Nice University Hospital, Nice, France

${ }^{7}$ Department of General Practice, Nice Sophia Antipolis University, Nice, France

Correspondence to Dr Cliodna McNulty; cliodna.mcnulty@phe.gov.uk

\section{ABSTRACT}

Objective To determine the needs of primary healthcare general practice (GP) staff, stakeholders and trainers to inform the adaptation of a locally successful complex intervention (Chlamydia Intervention Randomised Trial (CIRT)) aimed at increasing chlamydia testing within primary healthcare within South West England to three EU countries (Estonia, France and Sweden) and throughout England.

Design Qualitative interviews.

Setting European primary healthcare in England, France, Sweden and Estonia with a range of chlamydia screening provision in 2013.

Participants 45 GP staff, 13 trainers and 18 stakeholders. Interviews The iterative interview schedule explored participants' personal attitudes, subjective norms and perceived behavioural controls around provision of chlamydia testing, sexual health services and training in general practice. Researchers used a common thematic analysis. Results Findings were similar across all countries. Most participants agreed that chlamydia testing and sexual health services should be offered in general practice. There was no culture of GP staff routinely offering opportunistic chlamydia testing or sexual health advice, and due to other priorities, participants reported this would be challenging. All participants indicated that the CIRT workshop covering chlamydia testing and sexual health would be useful if practice based, included all practice staff and action planning, and was adequately resourced Participants suggested minor adaptations to CIRT to suit their country's health services.

Conclusions A common complex intervention can be adapted for use across Europe, despite varied sexual health provision. The intervention (ChlamydiA Testing Training in Europe (CATTE)) should comprise: a staff workshop covering sexual health and chlamydia testing rates and procedures, action planning and patient materials and staff reminders via computer prompts, emails or newsletters, with testing feedback through practice champions. CATTE materials are available at: www.STltraining.eu.

\section{INTRODUCTION}

Across Europe, the incidence of infection and transmission of genital Chlamydia
Strengths and limitations of this study

- The main strength of this study is that it gives public health leads the practical information they need to adapt and use a successful intervention that significantly increased chlamydia testing in a single area in England to other areas across Europe.

- The study included European Union (EU) countries that varied in their chlamydia control programmes showing that this needs assessment can be applied across European countries.

- The study included a range of different general practice staff, trainers and primary care public and sexual health stakeholders gaining a wide range of opinions about facilitators and barriers to delivering sexual health services and training in primary care across four countries.

- The study could have included more participants from each country to enrich the data and possibly find more facilitators or barriers to the intervention; however, the data were very consistent, and data saturation was reached in each country.

- To facilitate implementation in other countries and areas, further needs assessment on local chlamydia testing and staffing provision may be needed to completely inform any further training modifications.

trachomatis (chlamydia) continues to rise. ${ }^{1}$ Despite EU recommendations for opportunistic testing of asymptomatic individuals, ${ }^{12}$ there is still substantial heterogeneity in chlamydia control activities across Europe, ${ }^{34}$ and rates of chlamydia vary widely, mainly due to lack of ascertainment. ${ }^{1}$ In $2012,61 \%$ of EU countries reported no national strategy or plan for sexually transmitted infection (STI) control, and only six had a specific strategy for chlamydia testing and diagnosis. Costs of consultation, diagnosis, treatment and partner notification for STIs were only fully covered or reimbursed in $39 \%$ of countries. ${ }^{3}$ This indicates that further activities 
and support are required to increase chlamydia testing in many countries.

To increase chlamydia testing rates across the EU, the general practice could play a greater role in testing, as two-thirds of young people visit their GP practice annually. ${ }^{5}$ Young people report that GP surgeries are an acceptable venue for chlamydia testing and, furthermore, they think it is the GP surgeries' 'duty' to offer it. ${ }^{5-7}$

In England, we previously developed a complex chlamydia intervention, termed Chlamydia Intervention Randomised Trial (CIRT), that aimed to increase chlamydia testing for young people in the general practice setting by influencing: personal attitudes of GP staff to offering chlamydia testing routinely to young people, social norms in the practice around chlamydia testing and opinions of their young patients about testing and perceived behavioural controls in the practice setting of general practice (GP) staff and their patients. ${ }^{8}$ The CIRT was based on the Theory of Planned Behaviour (TPB). TPB is a psychological behaviour change theory that helps researchers to understand an individual's behaviour. The TPB can also be used to inform intervention development. ${ }^{9}{ }^{10}$ In this setting, the behaviour was GP staff offering a chlamydia test to all young people 16-24 years in the general practice setting. The intervention aimed to positively influence personal attitudes (GP staff emotional reactions towards chlamydia testing in the practice and beliefs that chlamydia testing makes a difference), subjective norms (positively influencing all practice staff and patients' beliefs about the value and ease of chlamydia testing in general practice and their motivation to undertake testing) and addressing real or perceived behavioural controls (staff and patients' perception of their ability (knowledge and skills) and degree to which various external factors (eg, time limitations) make it easy versus difficult to test all young people) (see online supplementary file 1 for constructs of the TPB).

The key components of CIRT included: an interactive workshop displaying the practice's chlamydia testing rates, the importance of and procedure for chlamydia testing, with examples of scripts to use in a consultation, and action planning; posters; patient leaflets; computer prompts; and ongoing feedback. In 2010, CIRT, in South West England, ${ }^{8}$ significantly increased chlamydia testing rates, and the effect was sustained. CIRT more effectively increased testing if GPs used computer reminders and participated in further feedback, with a chlamydia support worker. ${ }^{8}$ Translation of CIRT's educational resources into other European languages, and adaptation where necessary, presents a real opportunity to increase testing in other countries, particularly where testing in general practice is low. Previous groups who have adapted public health interventions for different EU countries ${ }^{11} 12$ highlight the importance of understanding the major differences in needs between the previous and future target groups and of maintaining the key elements of the intervention. ${ }^{12}$
This paper describes the qualitative needs assessment used to determine the needs of primary healthcare general practice staff, stakeholders and trainers to inform the adaptation of CIRT for a wider primary healthcare audience within England and three EU countries that vary in their chlamydia control programmes (Estonia, France and Sweden), so creating ChlamydiA Testing Training in Europe (CATTE).

\section{METHODS}

\section{Setting}

The needs assessment was undertaken by five centres in four countries: England, within the primary care unit and National Chlamydia Screening Programme (NCSP) of Public Health England; Estonia, within the Department of Public Health at the University of Tartu; Sweden, within the University Hospital, Örebro; and France, within the Nice University Hospital Département de Santé Publique, Nice. The assessment and resource development were undertaken by a multidisciplinary team including microbiologists, public health and primary care researchers and clinicians.

\section{Participants}

In 2013, in order to obtain a sample of GP staff working in small and large rural and urban practices, involved in training students or not, of different gender and range of age, we aimed to interview at least eight GP staff in each partner country. In Estonia, France and Sweden, GP staff were approached purposively to fulfil these criteria, using the routine existing GP practice contacts list used by the institution. In France, where doctors work singlehandedly, more individuals were interviewed to obtain more opinions. In England, where the modified intervention would be piloted across the country, we approached GP practices in three areas, which were inner city London, rural and urban (Islington, Warwickshire and Bournemouth). English practices were ranked by 2012 chlamydia screening rates; six practices in the highest tertile in each area were approached in random order. We then purposively selected six practices with low chlamydia testing numbers that were closest geographically to these high testing practices.

To ensure the training was appropriate, feasible and fitted in with local sexual health policy, future trainers who would be involved in intervention delivery and four stakeholders involved in the provision or commissioning of local public and sexual health services were purposively selected and interviewed. Trainers were nurses, general practitioners or public health physicians with some knowledge of sexual health but not specialists.

\section{Interview schedule}

All researchers worked together to create semistructured interview schedules based on the TPB (see online supplementary file 2). The schedule covered participants' (GP staff, stakeholders and trainers) own and perception of 
others' views on the importance, perceived effectiveness and current practice of offering chlamydia testing, condoms, contraception and sexual health services in general practice and GP staff's confidence and other external factors influencing their ability and intention to do so. We also guided all participants through the contents of the English CIRT, asking their opinions about each component and its delivery. The interviews also explored the optimum training models in each country. The interview schedule was piloted with four GP staff in England, with alterations made after the first two by the English researchers and repiloted with two more GP staff. Only minor changes were made after this schedule was piloted and with one or two GP staff in each of the other countries. Potential participants were approached by email or phone through the practice managers, and interviews were conducted face to face in a private room in the GP practice, lasting between 11 and 57 mins. In Sweden, interviews were also conducted in the county clinical microbiology laboratory (see online supplementary file 3 recruitment flow charts). Interviews in each country were in their native language and slightly modified to allow for any differences in the focus of sexual health education requested by stakeholders. Interviewers were practiced in the skill of interviewing, using probing as opposed to leading questions. The researcher made field notes of salient themes and main findings, during and after the interview.

\section{Analysis of interview data}

Interviews were audio-recorded, transcribed in the native language and cross- checked for accuracy by researchers against the recordings. Participants were able to request to comment on the transcript if they wished; none did so. All data were analysed using the same thematic analysis. ${ }^{13}$ Immediately after individual interviews, interviewers summarised key themes to inform the analysis coding frame. Two researchers in each country identified and agreed initial and strong themes. England and France used QSR NVivo V.10 software to organise data into themes; other countries used Excel spreadsheets. After the researchers in each country had undertaken at least several interviews and agreed initial themes, a high level thematic and coding framework was agreed by all researchers at a teleconference. The framework of themes was based on the initial findings of each country (table 1). The agreed coding frame was used to research each country's data but researchers were able to add more themes as they arose.

A second teleconference allowed further discussion of additional themes arising in each country, so these could be explored in the data of other countries. A final coding frame was developed by each country, and quotations, which illuminated the final themes, were exported into Microsoft Word and translated into English and checked by a second researcher in each country. The final results were presented by each country at a face-to-face meeting. Researchers discussed how the barriers to chlamydia testing identified could be overcome by the facilitators suggested by participants and used to adapt the English CIRT's materials and optimise the intervention.

\section{RESULTS}

Across the four partner countries, a total of 45 GP staff, 18 stakeholders and 13 trainers were interviewed, reaching data saturation ${ }^{14}$ (England 25, Estonia 15, France 23 and Sweden 13). In the results, if GP staff, trainers and stakeholders all reported the same theme, they are referred to as 'participants'. See online supplementary file 4 for full quotes supporting main themes.

\section{Existing chlamydia testing in general practice}

Although there was sometimes one member of GP staff with sexual health interest, who considered that general practice was an ideal chlamydia testing venue,

Table 1 Initial broad thematic framework agreed by partners

\begin{tabular}{|c|c|c|}
\hline Personal attitudes & Subjective norms & Behavioural controls \\
\hline Role of GP practice in testing for STIs & $\begin{array}{l}\text { Value and practicalities of involving all } \\
\text { GP staff }\end{array}$ & $\begin{array}{l}\text { Staff knowledge and skills around testing } \\
\text { procedure }\end{array}$ \\
\hline Benefits of testing for chlamydia & $\begin{array}{l}\text { Testing practice in the area and } \\
\text { guidelines }\end{array}$ & $\begin{array}{l}\text { Patient knowledge around testing } \\
\text { procedures }\end{array}$ \\
\hline $\begin{array}{l}\text { Attitude about raising sexual health } \\
\text { chlamydia in different consultations }\end{array}$ & Patient knowledge and attitudes & Attendance of young people at surgery \\
\hline $\begin{array}{l}\text { Perceived views of young people around } \\
\text { chlamydia testing }\end{array}$ & Types of trainer that would be respected & $\begin{array}{l}\text { Facilitators: for example, computer } \\
\text { prompts, reimbursement/ } \\
\text { Testing process and materials needed in } \\
\text { the practice }\end{array}$ \\
\hline
\end{tabular}

GP, general practice; STI, sexually transmitted infection. 
no country reported chlamydia testing as normal practice by all GP staff, and most participants (GP staff, trainers and stakeholders) reported that testing in asymptomatic patients was not routine, especially if the consultation was unrelated to sexual health. Even in England, with an established chlamydia screening programme, ${ }^{15}$ GP staff reported little opportunistic chlamydia screening (box 1).

\section{Box 1 Existing culture and infrastructure of chlamydia testing in general practice.}

\section{No culture of testing patients for chlamydia as normal practice.}

Asymptomatic Chlamydia screening?... None really! I only ask (about) a chlamydia test when I suspect a STI, in that case I always add chlamydia. (France, GP staff)

\section{Views on combining chlamydia testing, condoms and signposting to contraception (3Cs) into a routine basic sexual health offer.}

Well, I mean, basically I just feel it's absolutely so important but I'm really a strong advocate of sexual health services being provided as part of a holistic [approach]. (England, stakeholder)

No problem. I could myself ask every young patients but I do not do so now. But that could be a possibility. I have no problem with that. (Sweden, GP staff)

Are there places where they can get free condoms? And where is that? (France, GP Staff)

We do not have time for screening and sexual health discussions if the patient do not ask for that. (Sweden, GP staff)

\section{GP staff do not generally ask about sexual health in an unrelated consultation.}

If a patient comes with UTI problems, then I feel it is relevant. If it is a sore throat, then I don't feel I would start offering chlamydia test. (Estonia, GP staff)

\section{Most staff in all countries reported a need for an update on chlamydia.}

We really have this knowledge on chlamydia and gonorrhea but we need to be reminded. That is good. (Sweden, GP staff)

I think it's an area that we need to address because we're very prudish in this country and you know I think we've got one of the highest teenage pregnancy rate. (England, GP staff)

STI training would be very relevant and interesting. I had very limited information about STIs in my residency and I would be very interested to have some training. (Estonia, GP staff)

We would need some training... being updated on the subject and helped to suggest screening, our patients would agree without problems! And we need the test kit of course and learn how to show patients to use it. (France, GP staff)

It's a little bit more difficult [offering chlamydia tests] with the boys because they don't come in, for any of that sort of stuff [sexual health]. Very rarely. (England, GP staff)

\section{Individuals aged 15-24 years do not attend GP surgeries, especially for sexual health.}

Patients are not aware that they can be tested for STIs at their GPs. Need to inform patients, as we do for example with PAP test. (Estonia, GP staff)

\section{Patients would be anxious about confidentiality.}

In Estonia, GP for a young person are chosen by their parents. That could be a problem. Out of fear, that certain information could reach parents, will make this young person go to Youth Counselling Centre [not their GP]. (Estonia, GP staff)

Young people prefer to go to the Youth Health Center for sexual discussions. They are anxious that the GP staff may say something to their parents. (Sweden, GP staff)

\section{GP staff perception that patients need to request a test.}

No, it's the patient at the moment [who needs to request a test]. (England, GP staff)

Those kind of things come primarily from the patient, not from the GP. (Estonia, GP staff)

\section{Value of patient information to increase patient awareness of chlamydia testing in general practice.}

'(Talking about the poster) I think the message you want to get across is that we (the surgery staff) are happy to test.' (England, GP staff) I would like to have informative poster in my office. (Estonia, GP staff)

A poster that can be seen by everyone will be helpful, and perhaps, the invitation cards as well, so the teenagers are a bit prepared for the question. And the poster is also a reminder for the staff. (Sweden, GP staff)

\section{Need for an information leaflet in France.}

Chlamydia is not very well known by the patients in general, so posters and leaflets would make it easier for us to raise their awareness! A card would be too direct, they wouldn't understand, they need more information... from scratch. (France, GP staff) 
Many GP staff thought that combining the offer of chlamydia screening with an offer of contraception and condoms was a very good idea, but most said that they would struggle to fit this all in within a normal consultation, without extra resources; some suggested it should just be offered in young persons' clinics. Stakeholders stressed that, if condoms were offered, they should be free to patients and practices. Most GP staff in all countries reported a need for an update on: sexual health and chlamydia, how to test, treatment and partner notification. This was particularly pertinent in Estonia and France, where GP staff reported very little testing.

Many GP staff perceived that they did not see many young patients at risk of chlamydia, especially young men. Trainers and stakeholders also reported that a few GP practices felt they did not need to offer chlamydia testing as 'their patients did not put themselves at risk'. Many GP staff reported that patients preferred to seek sexual health services elsewhere, as patients were anxious about confidentiality, or sexual health services were not readily available in GP practices. There was a common GP staff belief in all countries that patients needed to ask for chlamydia testing, rather than staff proactively offering it; they suggested that posters would facilitate the offer of testing. All participants reported great value in providing young people information about surgery sexual health services, as posters, invitation cards, leaflets or website information. In France, participants reported that patients needed a detailed leaflet, as they knew less about chlamydia. A few GP staff, with a diverse population, stressed the importance of providing information in different languages.

\section{BARRIERS TO CHLAMYDIA TESTING IN GENERAL PRACTICE}

Time and pressures of other waiting patients were cited by all participants in each country as the key barrier to GP staff offering chlamydia testing and sexual health services. Asking about chlamydia testing alone would be difficult; asking about chlamydia, contraception and condoms together was perceived as even more difficult, although considered desirable by many. Most participants agreed that this time barrier could be decreased if GP staff were taught how to make the offer using short scripts of common consultations, demonstrated with written scripts (all countries), videos (England and Estonia) or role play (in Estonia) (box 2).

Forgetting to make the offer was common in all four countries. GP staff in England suggested that computer prompts or templates could be helpful to develop a consistent approach to chlamydia testing, enabling all staff, including receptionists, to be involved. Although some participants in other countries thought computer prompts were a good idea, implementation would be too complex due to the many different GP computer software systems.

In Sweden, as GP staff were paid for all chlamydia tests, they could undertake tests whenever it was clinically indicated or patients requested them, if they had time. Other countries' GP staff and stakeholders cited that limited practice funding for chlamydia testing meant that they were less likely to undertake this work, compared with other paid for public health interventions. A few GP staff in England reported that, where a locally enhanced service (LES) payment system, in which practices were given extra payments for submitting more chlamydia tests in young patients, had previously been in place and GP staff were already in the routine of asking about screening, screening had continued, despite the funding being withdrawn.

In Sweden, because diagnostic staff were available in each practice, arranging chlamydia testing was reported to be much easier by GP staff and stakeholders. In all other countries, many GP staff were not completely aware of the exact specimen collection procedures and the availability of self-sampling. As some male GP staff were not aware of self-sampling, they reported that they would need a female chaperone in the room, if testing a woman for chlamydia, and this made the testing procedure more time consuming while they arranged this. Most GP staff were enthusiastic about self-taken vaginal swabs, but some in all countries thought that asking women to do a vaginal swab would be more difficult than urine. A few GP staff raised the issue that they did not know if the patient would actually return the test once they picked up the kit, and several suggested that doing the test there and then may increase tests returned. When suggested to other GP staff, this testing 'there and then' approach sometimes raised time and practicality barriers. In France, patients cannot complete the test within their GP practice; they have to take the test to a laboratory with a GP prescription and their health insurance card to get the test analysed and be reimbursed for it. French GPs suggested that a patient information leaflet would help to increase patient awareness of this complex process.

\section{Workshop delivery}

All stakeholders agreed that, as in CIRT, chlamydia testing should be the central component of the CATTE intervention. In England, stakeholders indicated that the educational workshop should also cover signposting and advice on contraception, the provision of free condoms and HIV testing to reduce late diagnosis of HIV. Stakeholders and trainers suggested that HIV testing should be incorporated in England as a second workshop (training was termed 3Cs \& HIV). ${ }^{16}$ In France, a comprehensive sexual health approach was suggested by stakeholders, encouraging dialogue between young patients and their GPs about sexual health. Stakeholders in Sweden suggested that gonorrhoea testing should be incorporated, as dual testing for C. trachomatis and Neisseria gonorrhoeae is recommended due to the increasing cases of gonorrhoea among young heterosexuals. Estonia stakeholders preferred to just cover chlamydia and did not wish to add any further sexual health topics (box 3 ). 
Box 2 Barriers to general practice (GP) staff offering and patients returning chlamydia tests

\section{Diagnostic staff present in GP practices in Sweden makes testing process easier}

It is easy to instruct the patients how to take the test. Urine sample for males, self-taken vaginal sample for women. They just take the sample in the toilet here. And they will have the result within a week. (Sweden, GP staff)

\section{Time}

I am afraid that there is not enough time for that... For doing that, you have to have the will, time and place. This can't be done routinely. (Estonia, GP staff)

Well, it would depend on how I am, in terms of timing, so I may not be able to do it [chlamydia tests or contraception] there and then if I'm running late. And if I've got patients waiting, that's not fair [to others] really... But I do appreciate that, by saying that, I could be losing someone who might not come back [for a chlamydia test]. (England, GP staff)

\section{Covering chlamydia, condoms and contraception (3Cs) is even more difficult within a consultation}

We do not have time for screening and sexual health discussions if the patients do not ask for that. (Sweden, GP staff) 3 Cs [chlamydia, condoms and contraception] is being pitched as three quick questions, but it's three quick questions that are on top of a consultation that you have already had, and time is a resource as well. My own experience is, I manage to fill my 10 min very readily with all the other things. (England, GP staff)

\section{Education, videos or scripts to show staff how to make the offer quickly}

(referring to videos or scripts) Yes, I think, I think it's important for our staff to know how to approach it, because it's a very delicate issue and I think it needs that sort of information on how to do it. (England, GP staff)

A video would be far too time consuming. The GP will be able to spend only a short time for the training in the practice. (France, trainer) I do not think a video that is produced in another country will have any effect on Swedish GP. Initial education will be good enough. (Sweden, stakeholder)

\section{Computer prompts or templates as reminders}

And often the best way to do is to actually incorporate it [chlamydia screening reminders] into a template. And then that way...you don't miss anything. (England, nurse)

I think it is [referring to computer prompts reminding staff to offer chlamydia screening to all 15-24 year olds] because once it comes up, it reminds the receptionists, and they don't have to think 'oh, is this patient in the right age group?'. If somebody selected that cohort patients and put it [the prompt] as a blanket on that. So that would cover everyone in that age range. That would be good. (England, nurse)

Computer prompts, that's too complicated in France. (France, GP staff)

\section{Financial remuneration for chlamydia tests}

I do have to stress, that the only way [this intervention] is going to work is if it has funding behind it, funding for the time, the extra time. (England, GP staff)

It is hard when we don't have any extra money for doing that. (Estonia, GP staff)

We have a lot of things to discuss with each patient. If we're asked to make 2500 different screenings, it's not possible within our $23 €$ consultations! (France, GP staff)

\section{Not sure of exact chlamydia testing process}

The kits, I haven't seen them for a [while]. There was one for men and there was one for women. If we had the kits, we would use them. (England, GP staff)

Testing STIs, GP needs special equipment. I usually advise to go to gynaecologist, so they can test for everything. (Estonia, GP staff)

\section{Doing self-taken swabs or urines in the surgery}

I think it's doable [to get patients to complete the chlamydia test immediately in the practice], but again, it depends on whether they are happy to do it here, whether there are time constraints... rushed, or if they will be happy to wait for the kit, and to do it, and drop it off... I think it would be. (England, GP staff)

It is no problem with self-taken vaginal swabs for young women or urine test for young men at our surgery. They collect the samples easily in the toilet, and give the sample to the laboratory personal. (Sweden, GP staff)

Most girls, you can persuade them to wee and send it off, but to persuade them to have swab is a different kettle of fish I think. (England, GP staff) I did not know that [self-taken swabs] was an option. It would certainly help. (Estonia, GP staff)

\section{Complexity of performing a diagnostic test in France}

Well, we could give the patients a kit with explanations about how to perform the test, but will they do it? That's another question. It's better to do it at home than here at the practice. (France, GP staff) 
Box 3 Delivery of the educational workshops and other suggestions to increase chlamydia testing

\section{Global sexual health approach}

A global sexual health approach seems more relevant. It's like the cardiovascular risk, if you target the cholesterol without speaking about smoking. So, speaking about Chlamydia, you also have to explain contraception [and] the other STIs. (France, stakeholder) I think the contraception offer has to be quite clear... The knowledge of GPS can vary enormously... I think a lot of, some GPs can still have some quite old fashioned ideas about contraception. (England, stakeholder)

\section{Delivery of educational workshop by trainer}

We need training from other GPS, it's really very different when you're a gynaecologist [rather than] a GP, [and] ideally at our practice. (France, GP staff)

This [workshop materials] is more for staff at Youth Health Centres [who deal most with young people's sexual health]. They [Youth Health Centres] have workshops and conferences every year both national and regional. It is of rather low priority for GP in general. (Sweden, stakeholder)

\section{Workshop timing}

An hour isn't a disastrous amount of time... If it was at a lunch time or whatever, then it's possible the others, the doctors, and perhaps the nurses will do it. (England, GP staff)

You may come to inform us at our regular information meeting at our surgery. You may have 45-60 min, no more. (Sweden, GP staff)

\section{Involving the whole general practice (GP) team}

By trying... a whole surgery approach. What I find is, its ok working with an individual; but, unless you can get the whole surgery to be, sort of, chlamydia positive... it doesn't work brilliantly. (England, trainer)

Each practice has individual needs

And it's different for each [practice] and it's trying to work out what's key for some and we've...now broken it down a bit strategically, in that we look at the practice, and we try and look at the individuals themselves... and work out what drives them and what their motivation [is]. (England, stakeholder)

\section{Academic detailing in France}

GPs generally don't have time to go to meetings, it's better to make an adapted presentation at the practice, an academic detailing visit, preferably peer-conducted, to be able to exchange experiences. (France, stakeholder)

\section{Case studies or role play}

I think you could give some case studies... Practical examples, you know, because that always makes people focus... I think that works when you're trying to convince people of the success of something. (England, stakeholder)

Information could be delivered in (a) more practical way, like discussing case studies, more about treatment and follow up. (Estonia, trainer)

\section{Views about use of newsletters and feedback on testing rates for GP staff}

I think it sounds great. I think that... I'm sure it would have an impact, and having that kind of follow up as well. (England, stakeholder) OK, no one wants to be the worst. (Sweden, GP staff)

\section{Involving receptionists}

We can give every young patient the small card for information, no problem, but we cannot discuss sampling here where everybody can listen. (Sweden, GP staff)

\section{Appointing a sexual health champion}

I think, also finding champions within each practice... if you find one lead within each practice... is then prepared to sell it to their colleagues then [it could work]. (England, GP staff)

\section{Young people respond to incentives}

We found that young people in this area respond, bizarrely enough, to sperms keyrings... 'Oh, ok, if you want a sperm keyring, go do a wee for me.' (England, trainer)

\section{Young people like to be texted their results}

They texted people and people really liked that... they got texted the results. (England, GP staff) 
GP staff indicated that workshops should be facilitated by experienced trainers, ideally by peers. Most participants agreed that workshops should be held in the practice. English and Swedish GP staff considered a workshop of only 1 hour would increase attendance. In Sweden, a stakeholder indicated that the content of the educational workshop was more suited for staff at Youth Health Centres' who deal most with young people's sexual health. Other trainers and GP staff indicated that, due to other practice priorities, it would be a real challenge to obtain training time within GP surgeries, but that persuading practice managers of the benefits of training would facilitate bookings and also help implementation of the intervention. French trainers and stakeholders suggested shorter one-to-one academic detailing for the single-handed GP staff; in Estonia, it was recommended that joint workshops with several practices would be more efficient and may help influence social norms, as the activity could be more of an area initiative and enthusiastic staff may be able to influence others.

Generally, trainers, stakeholders and GP staff were happy with the range of proposed materials used in CATTE. Participants agreed the need for a whole team approach but thought it would be very difficult to ensure full team attendance. Participants emphasised the importance of individualising the educational workshops for each GP practice and determining how identifying an individual as the sexual health champion in each practice could influence the staff team, converting the postworkshop enthusiasm into an increase in testing. A few GP staff in England and Estonia suggested that discussion of case studies or role play would be appropriate to increase staff confidence to offer screening. Staff capacity to provide the educational workshops and follow-up, and funding of them, was identified by stakeholders as a barrier to implementation, on a significant scale.

\section{Other suggestions to increase chlamydia testing}

GP staff's opinions on whether non-clinical staff, such as receptionists, should be involved in offering chlamydia testing were very dependent on the surgery waiting area. If it allowed confidential discussions, often receptionists could give out invites and kits; however, for the majority, confidentiality was an issue. Trainers highlighted the need for GP manager and receptionist training, so that they could understand the importance of chlamydia testing. In Sweden, stakeholders reported that diagnostic staff working in the practice could offer tests, and therefore they should be invited to workshops.

A few GP staff reported that: a sexual health lead within each practice, and an informative website, could facilitate testing and feedback. Other GP staff suggestions included: offering young people token gifts for returning a test and texting results.

\section{DISCUSSION}

Findings across the four countries were similar, and as each country only suggested minor adaptations to suit their country's sexual health service, this supports adaptation of the English training model, for other countries as CATTE. Using the TPB constructs allowed us to determine the factors influencing GP staff intention to offer chlamydia testing in the general practice and make sure they were addressed in the CIRT intervention, when modified for wider use. The negative attitudes of some GP staff and the influence of their negative colleagues' attitudes had a major impact on their intentions to undertake chlamydia testing. GP staff in many practices did not consider that this work was of high-eno ugh priority in the little time they had to see patients, and most did not wish to raise it in a consultation unrelated to sexual health. The CIRT workshop aims to increase positive attitudes towards testing, by stressing how common the infection is, how testing can reduce spread, morbidity and long-term complications and that patients want GP staff to provide the offer. Feeding back testing rates and newsletters will also help to influence these attitudes. Stakeholders stressed the importance of having key practice staff attending the workshop; this will require workshop facilitators to check which practice staff are most influential and confirm they can attend. There were many behavioural controls identified in all countries; knowledge and skills around the exact testing process and how to maximise sample return was lacking in all countries and needs to be covered in all workshops. Finally, time and forgetfulness were problems in all countries and encouraging the use of computer prompts, if possible, with easily accessible testing kits, should be encouraged. The scripts already present in the CIRT materials will help the GP staff to develop skills to offer tests more quickly. The testing procedure did vary in different countries, which will need addressing in the training. In Sweden, there were diagnostic staff in the practice, whereas in France the procedure was much more complex and will be a more difficult external barrier to overcome.

\section{STRENGTHS AND LIMITATIONS}

We included countries that varied in their chlamydia control programmes from little testing in primary care in France to an established NCSP in England. ${ }^{1}$ We spoke to different GP staff, trainers and stakeholders, gaining a wide range of opinions about facilitators and barriers to delivering sexual health services and training in primary care across four countries. However, to facilitate implementation in other countries, further needs assessment may be needed to inform any further training modifications in that country. The study could have included more participants from each country to enrich the data and possibly find more facilitators or barriers to the 
intervention; however, the data was very consistent and we did reach data saturation in each country. To facilitate consistency of data analysis, we used a common thematic framework; this may have biased researchers towards finding common themes; however, we do not think this is so as several themes were only found in single countries, and countries produced representative quotes for the themes they described.

\section{Other work in this area}

It is interesting that the barriers to chlamydia testing in general practice we identified in this study are similar to those identified across primary care settings: in England $^{1718}$ and Australia. ${ }^{19} 20$ Continued barriers to sexual health provision in general practice indicate that behaviourally based interventions are needed to allow chlamydia testing to become embedded in routine practice. GP staff in the previous CIRT study who successfully increased their practice's chlamydia screening ${ }^{21}$ had implemented many of the suggestions made by staff in the other countries of this study, including: computer prompts that helped staff remember to make the offer, having a designated lead and feedback about the general practice's testing rates. In CIRT, ensuring patients' collected samples when still in the general practice was not attained in most general practices, ${ }^{21}$ and this was seen as challenging in this study. This aspiration will be difficult for most practices, unless the process is very clear, and kits are readily available. Australian primary care nurses indicated that the provision of training, streamlining chlamydia testing and simplification of pathology ordering processes would also facilitate nurses' chlamydia testing. ${ }^{19}$ As other work has found little significant difference in GP care provision in our four countries involved, ${ }^{4}$ the intervention should be easily transferable. In Sweden and England, sexual health is established in the GP medical training curriculum, ${ }^{4}$ which will facilitate implementation, whereas in France, sexual health training was not mandatory and in Estonia, it was extremely limited. ${ }^{4}$

All GP staff outside of Sweden cited financial pressures as a major barrier. Although fiscal incentives have been proposed to positively impact on the personal attitudes and subjective norms of GP staff, ${ }^{22}$ other evidence indicates that payments alone do not increase chlamydia testing rates. ${ }^{23}$ Financial incentives may not be the primary driver of increased chlamydia testing during interventions that also use support, education and performance feedback. ${ }^{24}$ In a review of 28 qualitative or mixed-methods studies exploring factors influencing chlamydia screening rates, facilitators included the normalisation of testing, the use of nurses and other practice staff, education and incentives. ${ }^{20}$ The review indicated that a greater study of facilitators was needed. We hope our work has added to this body of evidence by confirming these successful facilitators and identifying other facilitators that could be used to increase chlamydia testing, including giving GP staff scripts to use to increase capability of offering tests, use of computer prompts or templates, patients undertaking the test immediately in the surgery before leaving the practice, peers as trainers and whole team approach including training receptionists.

\section{Implications}

This needs assessment indicated that CIRT could be adapted to address the needs of GP staff in four EU countries with a range of chlamydia testing provision in general practice. As a result of the CATTE project, the group has developed a training programme in each country, with components that address each of the facilitators and barriers reported in this needs assessment. ${ }^{16}$ Our findings indicate that any such workshop programme will need appropriate funding, feedback and ongoing support. It would be valuable for future evaluations to use a multifactorial design that includes financial incentives only in one arm.

Acknowledgements We wish to thank the National Chlamydia Screening Programme (NCSP) and wider project group in England, especially Kevin Dunbar, John Saunders, Anthony Nardone and Thomas Hartney, and all members of the 3Cs \& HIV Project Group (Deborah Shaw and Sharron Ainslie). Also, the 3Cs \& HIV Advisory Group in England (Sebastian Kalwij, Farah Paruk, Farhana Lockhart, Jane Wilkinson, Jason Warriner, Richard Ma, Suzie Clements and Vicki Courage). We wish to thank Kaia Enni and Tatjana Meister from Estonia. We thank Anne Lennell, Timmy Leijen, Johan Hedlund and Johanna Haraldsson for important contributions in the adaption of the Swedish model of CATTE. We wish to thank Sanne Veringa and Soline Detanne for their important work on the CATTE project in France. We also wish to express our thanks to the CATTE Project Audit Committee (Karin Haar, Chris Foy, Ruth Lowbury, Olga Rostowska and Torsten Berglund). We thank all the GPs, patients and stakeholders who were involved in the participating countries.

Contributors CM and EJR had been involved in the CIRT project. CM and EJR wrote the grant application and designed the study/project and wrote the draft interview schedules and protocols for discussion. HF, CR, KT, AU, AT-S, AM, KF, BD and PT discussed and agreed the protocols and interview schedule, were involved in adapting interview schedules, conducting and analysing of interviews and meetings in their countries and commented on the paper. In England, EJR and KT (both female) conducted the interviews, AM (female) conducted the interviews in France, HF (male) conducted the interviews in Sweden and AU (female) conducted the interviews in Estonia. RA was involved in analysing the English interview transcripts. All authors contributed to the resource adaptation process. CM and EJR wrote the manuscript with contributions from all authors. All authors have reviewed and approved the final manuscript.

Funding This study was supported by a Leonardo Da Vinci Lifelong Learning programme project Chlamydia Testing Training in Europe (number2012-1GB2-LE005.08044) and Public Health England.

Competing interests None declared.

Patient consent Obtained.

Ethics approval In England, local research and development approvals were obtained for the qualitative interviews from NHS Islington (Ref- CSP120435), Warwickshire Primary Care Trust (Ref- WAR201212), Dorset CCG (Ref- 13/ $\mathrm{EM} / 0018)$. No approvals were needed in the other three countries. All participants gave informed consent for interviews to be recorded, transcribed and for anonymised quotes to be used in publications.

Provenance and peer review Not commissioned; externally peer reviewed.

Data sharing statement The anonymised datasets generated and/or analysed during the current study are available from the corresponding author on reasonable request.

Open Access This is an Open Access article distributed in accordance with the Creative Commons Attribution Non Commercial (CC BY-NC 4.0) license, which permits others to distribute, remix, adapt, build upon this work non-commercially, and license their derivative works on different terms, provided the original work is 
properly cited and the use is non-commercial. See: http://creativecommons.org/ licenses/by-nc/4.0/

(C) Article author(s) (or their employer(s) unless otherwise stated in the text of the article) 2017. All rights reserved. No commercial use is permitted unless otherwise expressly granted.

\section{REFERENCES}

1. European centre for disease prevention and control. Annual epidemiological report 2014 - sexually transmitted infections, including HIV and blood-borne viruses. Stockholm, 2015.

2. Nwokolo NC, Dragovic B, Patel S, et al. 2015 UK national guideline for the management of infection with Chlamydia trachomatis. Int $J$ STD AIDS 2016;27.

3. van den Broek IV, Sfetcu O, van der Sande MA, et al. Changes in chlamydia control activities in Europe between 2007 and 2012: a cross-national survey. Eur J Public Health 2016;26:382-8.

4. Uuskula A, Ricketts EJ, Rugman C, et al. Provision of chlamydia testing, and training of primary health care staff about chlamydia testing, across four European copinions survey Report No. 41: contraception and sexual heath, 2008-2009 Office for national statistics countries. BMC Public Health 2014.14:1147.

5. Salisbury C, Macleod J, Egger M, et al. Opportunistic and systematic screening for chlamydia: a study of consultations by young adults in general practice: Br J Gen Pract 2006;56:99.

6. Lader D. Contraception and sexual heath, 2008-2009 office for national statistics. NHS Information Centre 2009.

7. Hogan AH, Howell-Jones RS, Pottinger E, et al. "they should be offering it": a qualitative study to investigate young peoples' attitudes towards chlamydia screening in GP surgeries. BMC Public Health 2010;10:616.

8. McNulty CA, Hogan AH, Ricketts EJ, et al. Increasing chlamydia screening tests in general practice: a modified Zelen prospective cluster randomised controlled trial evaluating a complex intervention based on the theory of planned behaviour. Sex Transm Infect 2014;90:188-94.

9. Hardeman W, Johnston M, Johnston D, et al. Application of the theory of planned behaviour in behaviour change interventions: a systematic review. Psychol Health 2002;17:123-58.

10. Steinmetz $\mathrm{H}$, Knappstein M, Ajzen I, et al. How effective are behavior change interventions based on the theory of planned behavior? $Z$ Psychol 2016;224:216-33.
11. McNulty CA, Lecky DM, Farrell D, et al. Overview of e-bug: an antibiotic and hygiene educational resource for schools. J Antimicrob Chemother 2011;66 Suppl 5:v3-v12.

12. Tortolero SR, Markham CM, Parcel GS, et al. Using intervention mapping to adapt an effective HIV, sexually transmitted disease, and pregnancy prevention program for high-risk minority youth. Health Promot Pract 2005;6:286-98.

13. Braun V, Clarke V. Using thematic analysis in psychology. Qual Res Psychol 2006;3:77-101.

14. Fusch PI, Ness LR. Are we there yet? data saturation in qualitative research. The Qualitative Report 2015;20:1408.

15. Public health England. Sexually transmitted infections and chlamydia screening in England, 2014. 2015.

16. Town K, Ricketts EJ, Hartney T, et al. Supporting general practices to provide sexual and reproductive health services: protocol for the $3 \mathrm{Cs}$ \& HIV programme. Public Health 2015;129:1244-50.

17. McNulty CA, Freeman E, Bowen J, et al. Barriers to opportunistic chlamydia testing in primary care. Br J Gen Pract 2004;54:508-14.

18. McNulty CA, Freeman E, Howell-Jones R, et al. Overcoming the barriers to chlamydia screening in general practice-a qualitative study. Fam Pract 2010;27:291-302.

19. Lorch R, Hocking J, Guy R, et al. Do Australian general practitioners believe practice nurses can take a role in chlamydia testing? A qualitative study of attitudes and opinions. BMC Infect Dis 2015;15:31.

20. Yeung A, Temple-Smith M, Fairley $C$, et al. Narrative review of the barriers and facilitators to chlamydia testing in general practice. Aust J Prim Health 2015;21:139-47.

21. Ricketts EJ, Francischetto EO, Wallace LM, et al. Tools to overcome potential barriers to chlamydia screening in general practice:qualitative evaluation of the implementation of a complex intervention. BMC Fam Pract 2016;17:33.

22. Scott A, Sivey P, Ait Ouakrim D, et al. The effect of financial incentives on the quality of health care provided by primary care physicians. Cochrane Database Syst Rev 2011;9:CD008451.

23. Bilardi JE, Fairley CK, Temple-Smith MJ, et al. Incentive payments to general practitioners aimed at increasing opportunistic testing of young women for chlamydia: a pilot cluster randomised controlled trial. BMC Public Health 2010;10:70.

24. Kalwij S, French S, Mugezi R, et al. Using educational outreach and a financial incentive to increase general practices' contribution to chlamydia screening in South-East London 2003-2011. BMC Public Health 2012;12:802. 